\title{
PIC code for the plasma sheath in large caesiated RF sources for negative hydrogen ions
}

\author{
D Wünderlich, R Gutser, U Fantz \\ Max-Planck-Institut für Plasmaphysik (IPP), EURATOM Association, \\ Boltzmannstr. 2, D-85748 Garching, Germany \\ E-mail: dirk.wuenderlich@ipp.mpg.de
}

\begin{abstract}
Powerful negative hydrogen ion sources are required for heating and current drive at ITER. The physics of production and extraction of high negative ion currents is much more complex than for positive ions. One of the most relevant parameters is the shape of the plasma sheath, which determines the velocity of surface produced negative ions and thus the probability of the ions to reach the extraction system. In order to investigate the influence of hydrogen atoms, positive and negative hydrogen ions and positive caesium ions on the plasma sheath, a 1d3v PIC code for the plasma close to the extraction system has been developed. For typical plasma parameters of such ion sources, surface conversion of impinging atoms is the main negative ion production channel, while conversion of positive ions plays a minor role. Due to the formation of a potential minimum close to the surface, the emission of negative ions into the plasma is space charge limited. As a consequence, the flux of negative ions can be increased only by increasing the density of positive hydrogen ions. At identical plasma parameters, an isotope effect is determined by the mass of the particles only, resulting in lower fluxes of negative deuterium ions compared to hydrogen. A small amount of positive Cs does not change the plasma sheath and the $\mathrm{H}^{-}$flux significantly.
\end{abstract}




\section{Introduction}

Heating and current drive in future fusion devices like ITER require neutral beam injection (NBI) systems based on negative deuterium ions [1]. The baseline design depends on an ion source which has to deliver a current of $40 \mathrm{~A}$, accelerated to $1 \mathrm{MeV}$ at a low source pressure $(p=0.3 \mathrm{~Pa})$.

The maximum current densities delivered by negative ion sources are typically by a factor of ten smaller compared to systems for positive ions. Thus, in order to fulfil the ITER requirements, a large source is needed (forseen is an extraction area of $0.2 \mathrm{~m}^{2}$ with an current density of $200 \mathrm{~A} / \mathrm{m}^{2}$ deuterium ions).

Recently, the $\operatorname{RF}\left(f_{\mathrm{RF}}=1 \mathrm{MHz}\right)$ driven high power $(P \approx 100 \mathrm{~kW}$ per driver $)$ negative ion source developed at IPP Garching $[2,3]$ was chosen as the new reference source for the ITER NBI system [4]. The production of negative ions in this source is based on the surface conversion process of hydrogen [5] or deuterium. Note: for convenience, in the following the term "hydrogen" means either hydrogen or deuterium, unless otherwise specifically mentioned.

The physics of production, transport and extraction of negative hydrogen ions is much more complex than for positive ions [6]. Understanding the mechanisms involved in ion sources with high extracted current densities is a key issue for optimizing the existing sources and supporting the construction of larger systems, for example the half-area ITER negative ion source test facility ELISE $[7,8]$ which is an intermediate step between present RF based negative ion sources and the ITER source.

As shown recently by transport code calculations [9], the plasma sheath that forms at the walls plays an important role for the extraction probability of surface produced negative hydrogen ions: the potential difference of the sheath determines the velocity of the ions and thus the rate coefficients of destructing events.

In principle, it is possible to determine the potential difference at the sheath by plasma diagnostics, even in the presence of perturbations such as RF oscillations [10]. However, such measurements suffer from an inability to distinguish between different possible causes of observed potential difference variations, for example surface and volume effects [11], pointing out the need for specific modeling efforts.

Previously, numerical simulations of plasma sheaths with negative ions have been carried out for volume production using Particle In Cell (PIC) codes [12, 13] and MonteCarlo transport codes [14]. On the other hand, analytic results on the plasma in the vicinity of Langmuir probes in multi-component plasmas have been published recently [15].

However, neither analytic nor numerical results on the plasma conditions, particle species and reaction mechanisms relevant to surface conversion based high power negative ion sources are available. Thus, a 1d3v PIC code with simplified geometry was developed to support systematic investigations on the influence of hydrogen atoms, negative hydrogen ions and positive caesium ions on the plasma sheath in negative hydrogen ion sources for ITER NBI. 
The paper first gives a brief introduction to the production of negative ions via the surface process. Next, the PIC code is described in detail. The results obtained for the IPP ion source $[2,3]$ as well as their consequences are discussed.

\section{Surface produced negative ions}

In large negative hydrogen ion sources with high extracted current densities the ions are produced predominately by surface production $[2,16,17]$. This production process is based on the transfer of electrons from the surface material to hydrogen atoms or protons approaching the wall:

$$
\begin{aligned}
& \mathrm{H}+\mathrm{e}_{\text {surface }}^{-} \rightarrow \mathrm{H}^{-}, \\
& \mathrm{H}^{+}+\mathrm{e}_{\text {surface }}^{-} \rightarrow \mathrm{H}, \mathrm{H}+\mathrm{e}_{\text {surface }}^{-} \rightarrow \mathrm{H}^{-} .
\end{aligned}
$$

In case of a preceding dissociation process, these reactions can also occur for molecules or molecular ions [18]. The first step in the conversion of protons is the neutralization of the proton which happens rapidly in some distance from the wall by either an Auger or resonant process [18]. After this first step, the surface production channels for protons and atoms are identical: with decreasing distance to the wall, the electron affinity level of the atom is shifted towards lower energies and additionally broadened [19]. If the electron affinity level lies energetically below the Fermi level of the surface, tunneling of an electron from the wall material to the atom is possible, resulting in the production of a negative ion.

The number of produced negative ions can be calculated from the number of impinging particles and the conversion yield $Y$ :

$$
\begin{aligned}
& \Gamma_{\mathrm{H} \rightarrow \mathrm{H}^{-}}=\Gamma(\mathrm{H}) \cdot Y_{\mathrm{H} \rightarrow \mathrm{H}^{-}}, \\
& \Gamma_{\mathrm{H}_{x}^{+} \rightarrow \mathrm{H}^{-}}=\Gamma\left(\mathrm{H}_{x}^{+}\right) \cdot Y_{\mathrm{H}_{x}^{+} \rightarrow \mathrm{H}^{-}} .
\end{aligned}
$$

A thin caesium layer $(\approx$ one mono layer) covering the walls reduces the surface work function to around $2 \mathrm{eV}[20]$ and thus significantly increases the efficiency of the surface production process. Normally, caesium is evaporated into negative ion sources from a reservoir, containing either liquid caesium [21] or a solid caesium compound [22]. During plasma operation almost the complete inventory of caesium in the plasma volume will be ionized due to the low ionization threshold energy of caesium $\left(\mathrm{E}_{\mathrm{th}}=3.89 \mathrm{eV}\right)[23]$.

The conversion yields depend on the surface work function and thus the wall material, the amount of impurities in and the thickness of the caesium layer. Theoretical expressions for the conversion yields have been derived in [19]. Experimentally determined yields are available for molybdenum surfaces with an optimal coverage of caesium $[18,24]$. The conversion yield for both production channels can reach values up to $25 \%$, depending on the particle energy.

The initial velocity of surface generated negative ions before acceleration in the plasma sheath depends on the wall material and the velocity of the impinging particle. In $[25,26]$ calculated energy reflection coefficients for hydrogen atoms impinging on 
different wall materials are presented. Additionally, it was shown that the initial direction of surface produced negative ions can be described by a cosine velocity distribution.

For electron temperatures higher than $\approx 2 \mathrm{eV}$, stripping of the negative hydrogen ion's additional electron that has a low binding energy $\left(E_{\mathrm{b}}=0.75 \mathrm{eV}\right)$ by collision with electrons $\left(\mathrm{H}^{-}+\mathrm{e}^{-} \rightarrow \mathrm{H}+2 \mathrm{e}^{-}\right)$is the main destruction channel. For lower electron temperatures other destruction channels take over. These are: mutual neutralization with protons $\left(\mathrm{H}^{-}+\mathrm{H}^{+} \rightarrow 2 \mathrm{H}\right), \mathrm{H}_{2}^{+}\left(\mathrm{H}^{-}+\mathrm{H}_{2}^{+} \rightarrow \mathrm{H}+\mathrm{H}_{2}\right)$ or $\mathrm{H}_{3}^{+}\left(\mathrm{H}^{-}+\mathrm{H}_{3}^{+} \rightarrow 2 \mathrm{H}+\mathrm{H}_{2}\right.$ or $4 \mathrm{H})$, associative detachment $\left(\mathrm{H}^{-}+\mathrm{H} \rightarrow \mathrm{H}_{2}+\mathrm{e}^{-}\right)$and collisional detachment with atoms $\left(\mathrm{H}^{-}+\mathrm{H} \rightarrow 2 \mathrm{H}+\mathrm{e}^{-}\right)$. In either case the rate coefficient depends only slightly on the ion temperature.

The source under investigation is based on the tandem concept of negative ion sources [27], in which a dense $\left(n_{\mathrm{e}} \geq 10^{18} \mathrm{~m}^{-3}\right)$ and hot plasma $\left(T_{\mathrm{e}} \geq 10 \mathrm{eV}\right)$ is generated and then cooled down to electron temperatures below $2 \mathrm{eV}$ by means of a magnetic filter field to minimize the ion destruction. As an additional effect of the magnetic filter, the electron density is reduced to around $10^{17} \mathrm{~m}^{-3}$.

Due to a short survival length of the negative ions (around a few $\mathrm{cm}$ ), predominately ions generated at the surface of the plasma grid (the first grid of a multi grid extractionsystem) can be extracted. These ions are initially accelerated towards the plasma volume by the sheath potential. To reach the extraction apertures the particle trajectories have to be deflected towards the extraction apertures by just the magnetic filter field and/or collisions.

The probability of ions reaching the extraction apertures depends strongly on their velocity and the magnetic field strength [9] since these parameters affect the ions path length inside the plasma and the collision frequencies for destruction events.

The velocity of the negative ions is determined mainly by the potential difference at the plasma sheath and thus is correlated with the density, the mass and the temperatures of all charged plasma particles. It is expected that due to the the presence of particles like $\mathrm{Cs}^{+}$and surface produced $\mathrm{H}^{-}$this potential difference will be considerably different than the sheath in two-component plasmas, for which simple analytic solutions [28] exist.

\section{The PIC code}

PIC codes [29] are based on the simultaneous calculation of the trajectories of many charged particles in a self-consistent manner. To reduce the CPU time needed, the number of plasma particles is reduced significantly by introducing so-called macro particles, each of which represents a fixed number of real particles. The trajectories of these macro particles are calculated by the equation of motion:

$$
\vec{F}=m \cdot \frac{\mathrm{d} \vec{v}}{\mathrm{~d} t}=q(\vec{E}+\vec{v} \times \vec{B})
$$




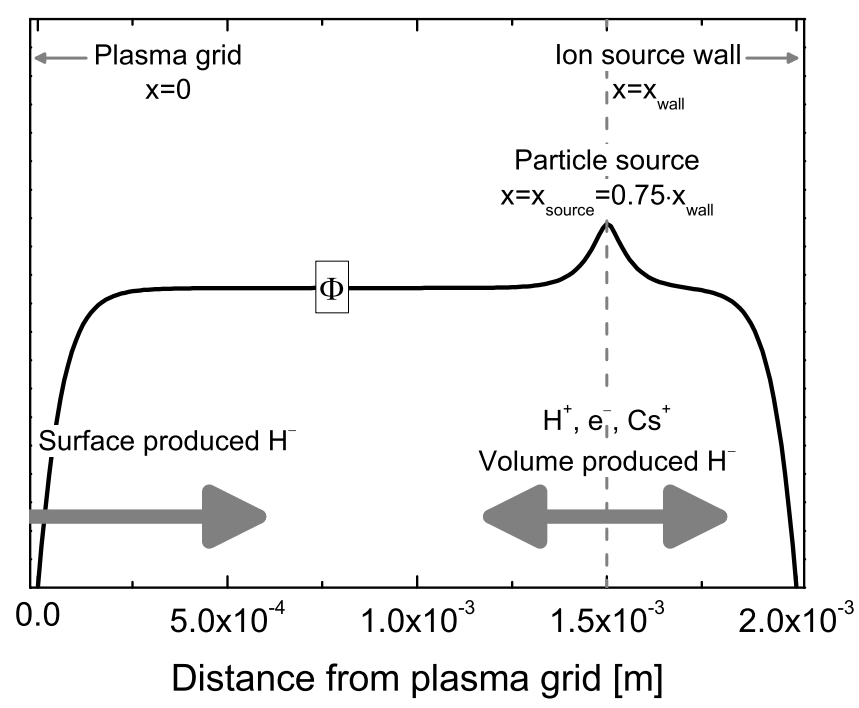

Figure 1. Calculation domain used for the $1 \mathrm{~d} 3 \mathrm{v}$ PIC code. The left and right boundary represent the plasma grid and a wall of the ion source, respectively. The plasma source inside the domain substitutes the bulk plasma.

The electric field $\vec{E}$ is calculated from the derivative of the electrostatic potential $\phi$, which is determined using Poisson's equation:

$$
\nabla^{2} \phi=-\frac{\rho}{\varepsilon_{0}} .
$$

The presented PIC code solves these equations by using the second-order accurate leap frog method [29] and the NAG [30] subroutine F11DBF, respectively.

The calculation domain is divided into grid cells. To avoid oscillations, the size of the grid cells and the calculation interval have to be chosen small enough to resolve the Debye length and the inverse plasma frequency, respectively [31]. Additionally, the Courant criterion [31] has to be fulfilled: the distance covered by the fastest particles within one time step must be smaller than the size of one grid cell. The space charge density, $\rho$, at the grid knots is determined by a linear weighting scheme.

Beginning with an empty calculation domain, for each time step the electrical field, the forces acting on the particles and the resulting particle trajectories are calculated. This procedure is iterated until an equilibrium is reached.

A 1d calculation domain was chosen which is shown schematically in fig. 1. The left boundary $(x=0)$ represents the plasma grid and the right boundary $\left(x_{\text {wall }}=2.0 \cdot 10^{-3} \mathrm{~m}\right)$ the grounded walls of the ion source. At both boundaries Dirichlet boundary conditions are used: fixed values of the potential.

The large bulk plasma volume is represented by the punctual particle source located at $x_{\text {source }}=0.75 \cdot x_{\text {wall }}$. This choice of $x_{\text {source }}$ results in a region of the size $\Delta x=1.5 \cdot 10^{-3} \mathrm{~m}$ between bulk plasma and plasma grid. In the case of a non-magnetized plasma sheath all physical effects relevant for the sheath formation take place in this region. In the presence of magnetic fields a larger calculation domain may be necessary (depending on 
the field strength) due to an increase of the sheath dimension [32]. At the position of the particle source equal numbers of positive ions $\left(\mathrm{H}^{+}\right.$and $\left.\mathrm{Cs}^{+}\right)$and electrons are injected each time step. The absolute numbers of injected particles are determined according to particle densities defined in the input to the code. $\mathrm{H}_{2}^{+}$and $\mathrm{H}_{3}^{+}$are neglected for the sake of simplicity, as no strong effect from either is expected. All three velocity components are included into the code. The initial velocity $v_{x}$ is determined randomly according to a Maxwellian flux velocity distribution function [33]:

$$
f\left(v_{x}\right)=\left|v_{x}\right| \cdot\left(\frac{m}{k_{\mathrm{B}} T}\right) \exp \left(\frac{-m v_{x}^{2}}{2 k_{\mathrm{B}} T}\right) .
$$

The initial velocities $v_{y}$ and $v_{z}$ follow ordinary Maxwell velocity distribution functions.

Negative ions produced at the surface by conversion of hydrogen atoms or conversion of positive hydrogen ions are introduced into the simulation domain at the plasma grid $(\mathrm{x}=0)$. When a positive ion hits the plasma grid, the conversion yield taken from [18] is calculated according to the particle energy. Then a Monte Carlo routine decides if a negative ion is produced or not. The conversion of neutral hydrogen atoms is implemented as an additional production process for negative ions based on the conversion yield from [24]. The rate of atoms hitting the walls is calculated from the atomic density as follows:

$$
\Gamma(\mathrm{H})=\frac{1}{4} \cdot\langle v(\mathrm{H})\rangle \cdot n(\mathrm{H})
$$

where the average impact velocity $\langle v(\mathrm{H})\rangle$ is calculated from the atomic temperature. A Monte Carlo routine is used to determine the velocities of the individual atoms impinging on the walls. Next the starting energy of the surface produced negative ions is determined according to: the energy of the incident particles, the energy reflection coefficient for a tungsten surface from [25] and a cosine velocity distribution.

Positive ions and electrons crossing the position of the particle source are started again with new random velocity and direction. This procedure ensures quasi-neutrality in the bulk plasma and represents thermalization of the particles by collision processes. Negative hydrogen ions which cross the particle source are destroyed. Around the particle source a so-called source sheath evolves which ensures quasi-neutrality in the calculation domain [28]. In real plasmas the source sheath spatially expands over the entire volume in which plasma particles are generated.

Collisions between particles are not considered because the size of the calculation domain is much smaller than the mean free path of electrons and ions. The plasma potential of the bulk plasma in the RF driven ion source under investigation varies over the time. Due to the fact that the plasma is inductively excited, no sheath collapse takes place during the RF phase; the amplitude of the plasma potential oscillations is small compared to the plasma potential itself and the oscillations can be averaged over time. Thus, this effect is neglected by the code and the results represent time averaged values. The magnetic filter field have not yet been included, since the focus is on understanding the basics of the sheath formation. To obtain smooth results, the profiles of particle 
Table 1. Plasma parameters used as input for the PIC code.

\begin{tabular}{lll}
\hline$T_{\mathrm{e}}$ & Electron temperature & $2 \mathrm{eV}$ \\
$n\left(\mathrm{H}^{+}\right)$ & $\mathrm{H}^{+}$density & $4 \cdot 10^{17} \mathrm{~m}^{-3}$ \\
$T\left(\mathrm{H}^{+}\right)$ & $\mathrm{H}^{+}$temperature & $0.8 \mathrm{eV}$ \\
$n(\mathrm{H})$ & $\mathrm{H}$ density & $10^{19} \mathrm{~m}^{-3}$ \\
$T(\mathrm{H})$ & $\mathrm{H}$ temperature & $0.8 \mathrm{eV}$ \\
$n\left(\mathrm{Cs}^{+}\right)$ & $\mathrm{Cs}^{+}$density & $10^{16} \mathrm{~m}^{-3}$ \\
$T\left(\mathrm{Cs}^{+}\right)$ & $\mathrm{Cs}^{+}$temperature & $0.8 \mathrm{eV}$ \\
\hline
\end{tabular}

densities and the potentials are averaged over several plasma oscillation periods after the equilibrium state is reached.

In order to benchmark the code, calculations for two-component plasmas at different ratios of electron to ion temperature were performed first. The calculated potential differences of source sheath and plasma perfectly agree with results of analytic calculations from [28].

Typical plasma parameters for a hydrogen plasma in the investigated negative ion source are shown in table 1 . Most of these values were determined by optical emission spectroscopy or Langmuir probe measurements [34]; the temperature of the positive hydrogen ions was estimated in [35], based on the Franck-Condon energy of hydrogen atoms $\left(E_{\mathrm{FC}}=2.24 \mathrm{eV}\right)$ and cooling by consecutive collisions with heavy particles. The temperature of positive caesium ions is assumed to be equal to the temperature of the other positive ions due to thermalizing collisions.

Calculations were performed for deuterium as well as for hydrogen. Although it is known that the plasma parameters differ for the isotopes, the calculations are based on the same set of plasma parameters. This simplification allows for focusing on the effect of the different particle masses on the plasma sheath, particle densities and fluxes.

The Debye length for these plasma parameters is $1.7 \cdot 10^{-5} \mathrm{~m}$ and the inverse plasma frequency is $2.8 \cdot 10^{-11} \mathrm{~s}$. Thus, the size of the grid cells and the calculation interval for the PIC code were chosen to be $5.0 \cdot 10^{-6} \mathrm{~m}$ and $5.0 \cdot 10^{-12} \mathrm{~s}$, respectively. Approximately 300000 time steps are needed $\left(1.5 \cdot 10^{-6} \mathrm{~s}\right.$ simulated time) to reach an equilibrium state. In this state the total number of macro particles present in the calculation domain is about $10^{6}$. Such a calculation needs less than a day on a $3.0 \mathrm{GHz}$ Xeon processor.

\section{Results}

In a parameter scan, the influence of the atomic density on the emission of negative hydrogen ions into the plasma volume is investigated over a wide range (between $10^{16} \mathrm{~m}^{-3}$ and $\left.10^{20} \mathrm{~m}^{-3}\right)$. Three regimes were identified. For low neutral densities conversion of protons (reaction 2) is the dominant surface production mechanism and thus the negative ion density is independent of the neutral density. With increasing atomic densities, the flux of atoms impinging on the plasma grid surface and thus the production of negative ions by conversion of neutrals (reaction 1) increases linearly and 


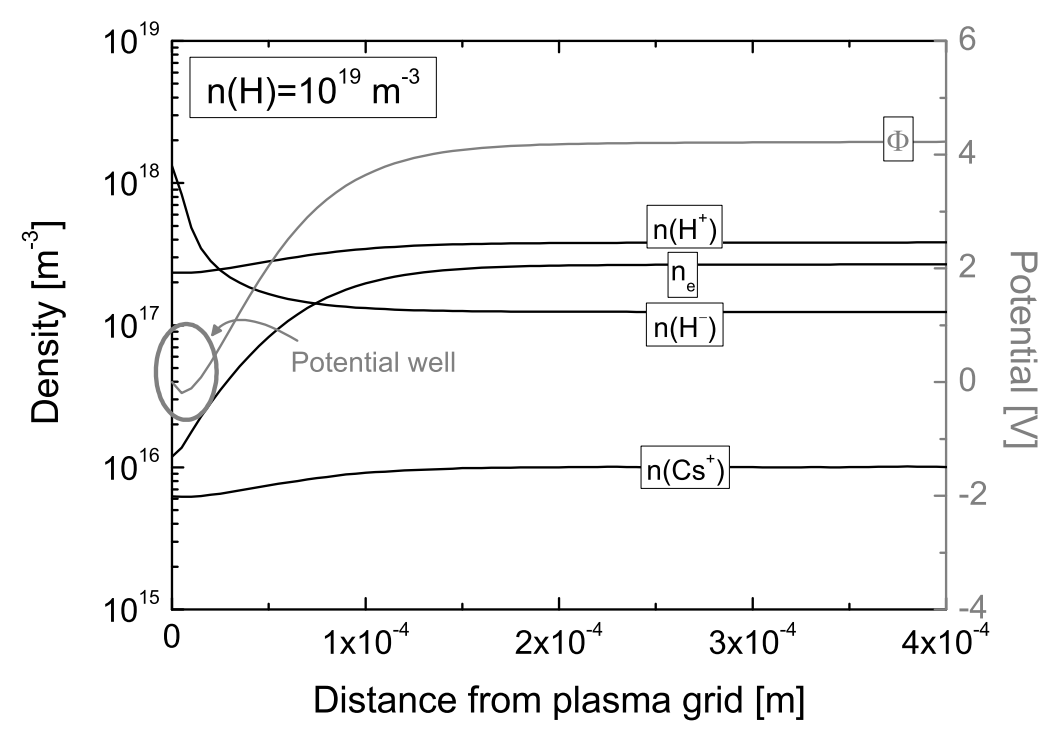

Figure 2. Density profiles and potential profile for typical plasma parameters of caesiated high power, high density negative ion sources.

gradually takes over. The increased negative ion production causes a strong negative space charge close to the plasma grid surface. Lastly, for high neutral densities, the emission of negative ions is negative space charge limited.

Figure 2 shows density and potential profiles calculated for the plasma parameters given in table 1 . The ratio of the negative ion density to the electron density is close to one in some distance from the wall $\left(n\left(\mathrm{H}^{-}\right) / n_{\mathrm{e}} \approx 0.45\right.$ for $\left.x>10^{-4} \mathrm{~m}\right)$. This result is in accordance with measured density ratios $[35,36,37]$ and indicates that the negative ions have a strong influence on the physics of the sheath region.

Total zero net current onto the grid is ensured by the plasma sheath, in which positive and negative particles are accelerated and repulsed, respectively. The potential difference of the plasma sheath $\left(\Phi_{\text {plasma }}-\Phi_{\text {grid }}=4.21 \mathrm{~V}\right)$ is about $1.17 \mathrm{~V}$ lower than in a pure electron-proton plasma with identical plasma parameters. This is due to the higher mass of negative hydrogen ions and positive caesium ions compared to electrons and protons and results in different accelerations of the charged particles crossing the sheath than in a two-component plasma.

Close to the plasma grid the above mentioned negative space charge can be seen, and the potential minimum it causes. The formation of this charge accumulation can be explained by the fluxes of negative ions generated by conversion of atoms:

$$
\begin{aligned}
\Gamma_{\mathrm{H} \rightarrow \mathrm{H}^{-}} & =4.36 \cdot 10^{21} \frac{1}{\mathrm{~m}^{2} \mathrm{~s}}, \\
\Gamma_{\mathrm{H}^{+} \rightarrow \mathrm{H}^{-}} & =1.09 \cdot 10^{21} \frac{1}{\mathrm{~m}^{2} \mathrm{~s}} .
\end{aligned}
$$

It can be seen that the conversion of atoms is the dominant of the two channels. The total flux of surface produced negative ions is too large to be removed from the area close to the grid surface within a sufficient time scale and thus the particles accumulate. 


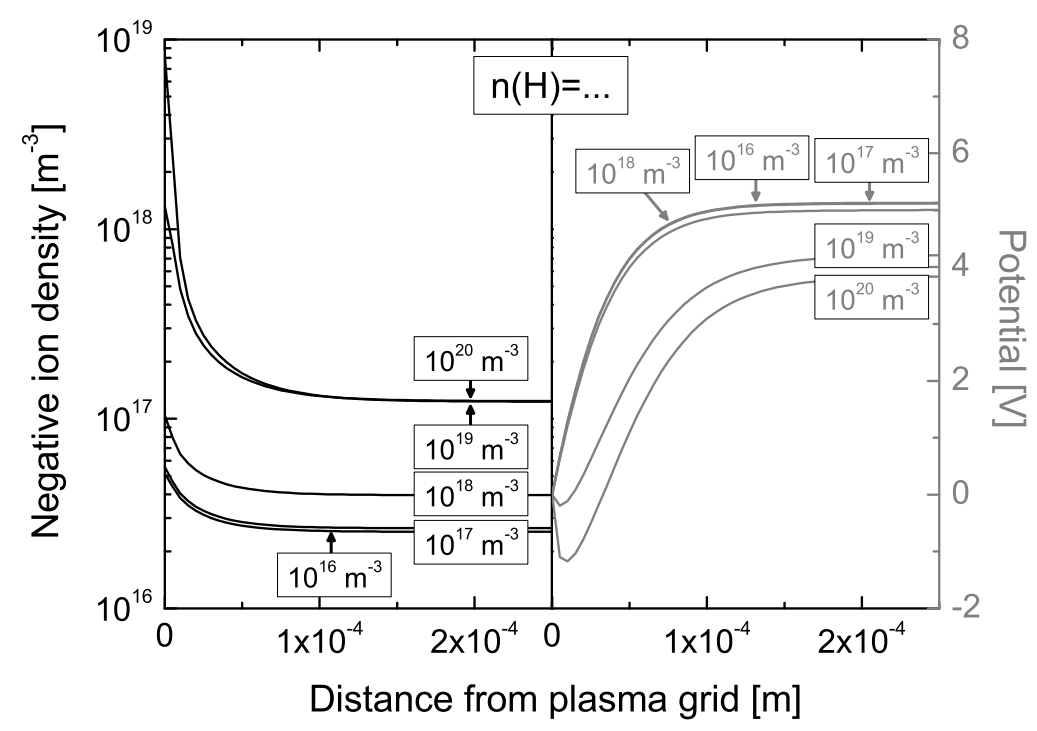

Figure 3. Close-up view of negative ion density profile and potential profile for typical plasma parameters of caesiated high power, high density negative ion sources and atomic densities between $10^{16} \mathrm{~m}^{-3}$ and $10^{20} \mathrm{~m}^{-3}$.

A certain fraction of the negative ions entering the potential well are reflected towards the surface, thus preventing a further rise of the negative ion density in this region.

With increasing atomic hydrogen influx the potential well deepens and the fraction of ions able to escape into the plasma volume decreases. This can be seen in figure 3 , where are shown the dependence of the potential and the negative ion density on the neutral density. For atomic densities smaller than $10^{18} \mathrm{~m}^{-3}$ no potential well is observed. For atomic densities larger than $10^{19} \mathrm{~m}^{-3}$ the increasing depth of the potential well directly shifts the plasma potential. In both cases the negative ion density in the plasma is independent of $n(\mathrm{H})$. The negative ion density depends directly on the hydrogen atoms only in the atomic density range between $10^{17} \mathrm{~m}^{-3}$ and $10^{19} \mathrm{~m}^{-3}$.

This space charge limitation of the negative ion emission for high atomic densities is similar to the emission of secondary electrons at a wall: it was predicted theoretically [38] and verified experimentally [39] that the number of emitted electrons does not increase for emission coefficients above a critical value.

Figure 4 shows the calculated flux of negative ions dependent on the neutral density for hydrogen and deuterium, for each case with and without the presence of positive caesium ions in the plasma. It can be seen that similar to the results presented above, for low atomic densities the negative ion flux is predominantly generated by conversion of positive ions, while for high atomic densities the conversion of atoms takes over. This results in an asymptotic behavior of the negative ion flux which is almost constant for atomic densities smaller than $10^{17} \mathrm{~m}^{-3}$ or larger than $10^{19} \mathrm{~m}^{-3}$.

Due to the mass difference, velocities for deuterium are smaller by a factor of $\sqrt{2}$ than for hydrogen. Consequently, the resulting negative deuterium fluxes are reduced by a factor around 1.4 compared to the fluxes in the case of hydrogen. This result does 


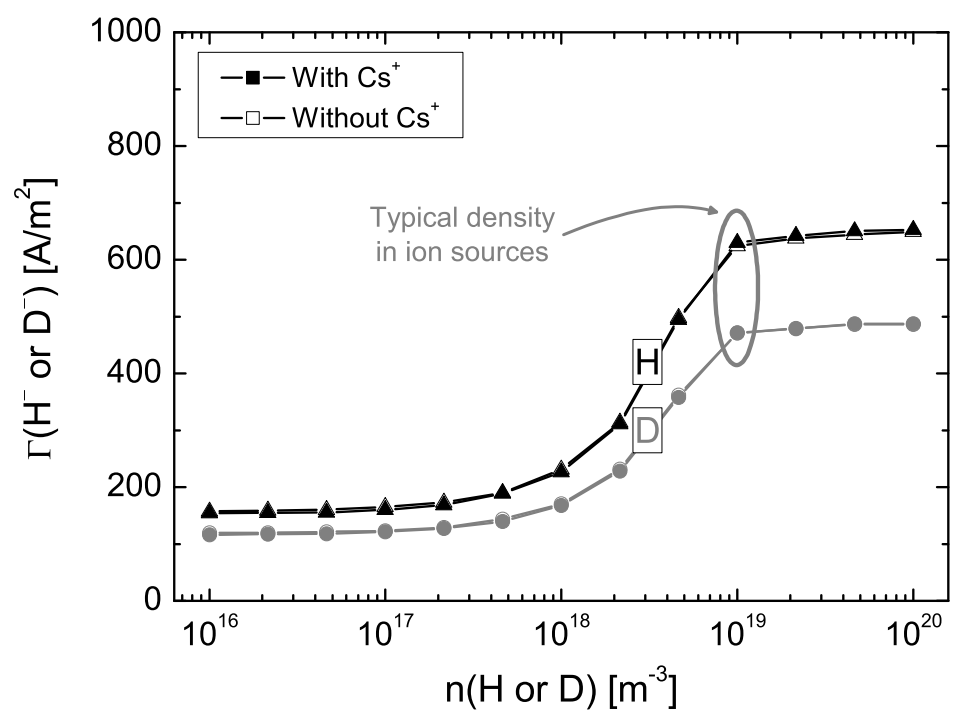

Figure 4. Comparison of $\mathrm{H}^{-}$and $\mathrm{D}^{-}$fluxes toward the plasma volume for typical plasma parameters of caesiated high power, high density negative ion sources. Calculations have been carried out for different values of the atomic density, with and without positive caesium ions. For both isotopes the same set of plasma parameters was used.

not agree with experimental results, where the source performance for both isotopes is almost identical [2]. Since it is known that the plasma parameters differ for the isotopes, this disagreement can be explained by the use of identical plasma parameters. The effect of the plasma parameters on the negative ion flux will be investigated in future.

The effect of the heavy caesium ions $\left(m\left(\mathrm{Cs}^{+}\right)=131.9 \cdot m\left(\mathrm{H}^{+}\right)\right)$on the plasma parameters and ion fluxes was studied by performing calculations without caesium ions in the plasma volume. To isolate the effect of caesium in the plasma volume from the surface effect, again an optimal caesium coverage of the surface was assumed, i.e. the conversion probabilities were not changed. Due to the absence of the, by far, heaviest particle species, the number of time steps needed by the code to reach equilibrium is decreased by a factor of three to approximately 100000 time steps $\left(5 \cdot 10^{-7} \mathrm{~s}\right.$ simulated time). Compared to figure 2 , the potential difference in the plasma sheath is lowered minimally by $0.04 \mathrm{~V}$ to $\Phi_{\text {plasma }}-\Phi_{\text {grid }}=4.17 \mathrm{~V}$. As a consequence of this smaller potential drop, the negative ion fluxes are slightly decreased. The relative decrease of the ion flux caused by the missing caesium ions is comparable for the two isotopes.

Additional calculations were performed for twice the density of caesium ions, i.e. $n\left(\mathrm{Cs}^{+}\right)=2 \cdot 10^{16} \mathrm{~m}^{-3}$. The potential difference is raised to $\Phi_{\text {plasma }}-\Phi_{\text {grid }}=4.27 \mathrm{~V}$ and only a slight increase of the negative ion flux is observed. This shows that for typical particle densities of the negative ion sources the influence of the caesium ions on the plasma sheath is small.

The calculated ion fluxes represent the number of negative ions leaving the plasma region around the plasma sheath. As shown in [9], the maximum extraction probability 
of negative ions produced at the surface of the LAG (Large Area Grid) extraction system [2] is $26 \%$, depending on the plasma parameters. Applying this value to the results of the PIC code for $n(\mathrm{H})=10^{19} \mathrm{~m}^{-3}$ yields an extracted ion current of around $170 \mathrm{~A} / \mathrm{m}^{2}$ with caesium ions. This value agrees with experimental results from a well conditioned ion source [21].

The space charge limitation of the negative ion flux indicates that for the present parameters the extracted current density can be further increased only by partially neutralizing the negative space charge, i.e. by increasing the positive ion density close to the grid surface. Additionally, the very slightly increased negative ion flux with caesium shows that the experimentally observed improvement of the source efficiency with caesium is mainly caused by the lowered surface work function.

\section{Conclusions}

Using a 1d3v PIC code, the plasma region close to the plasma sheath of a caesiated high power, high density negative ion source was investigated. Focus was laid on the potential difference at the sheath and the resulting negative ion densities and fluxes. For the first time, positive caesium ions and surface produced negative hydrogen ions were incorporated into the calculations. The calculations are based on plasma parameters from measurements in these sources.

Surface production of negative hydrogen ions can take place via the bombardment of a surface with sufficiently low work function by either atoms or positive ions. For the considered plasma parameters, conversion of $\mathrm{H}$ at the caesiated walls is the dominant of these two channels. The generation of $\mathrm{H}^{-}$at the walls causes an additional negative space charge close to surface to form, which results in the formation of a local potential minimum. The depth of this potential minimum increases with the amount of negative hydrogen ions present at the wall. As a direct consequence, the negative ion flux from the plasma grid surface into the plasma volume is space charge limited. This means that above a critical value of the atomic influx increasing this influx does not increase the flux of negative ions.

Studies on the isotope effect for deuterium at the same plasma parameters showed that the fluxes of negative ions compared to the results of hydrogen are smaller by roughly the ratio of the particle velocities $(\sqrt{2})$.

A small amount of positive Cs ions $\left(n\left(\mathrm{Cs}^{+}\right)<0.05 \cdot n\left(\mathrm{H}^{+}\right)\right)$slightly deepens the potential difference of the plasma sheath. The influence on the $\mathrm{H}^{-}$flux is negligibly small.

Future calculations will focus on the isotope effect by using different plasma parameters for deuterium. Additional effects to be investigated in detail are the effect of: variations of the plasma parameters, biasing of the plasma grid with respect to the source walls and the influence of the magnetic filter field. 


\section{References}

[1] ITER Technical Basis 2002, ITER EDA Documentation Series No 24 (Plant Description Document, section 2.5.1), IAEA Vienna.

[2] E. Speth, H. D. Falter, P. Franzen, and U. Fantz et al. Overview of the RF source development programme at IPP Garching. Nucl. Fusion, 46:S220-S238, 2006.

[3] P. Franzen, H. D. Falter, U. Fantz, and W. Kraus et al. Progress of the development of the IPP RF negative ion source for the ITER neutral beam system. Nucl. Fusion, 47:264-270, 2007.

[4] R. S. Hemsworth, A. Tanga, and V. Antoni. Status of the ITER neutral beam injection system (invited). Rev. Sci. Instrum., 79:02C109-1-02C109-5, 2008.

[5] K. N. Leung, S. R. Walther, and W. B. Kunkel. $\mathrm{H}^{-}$Formation in a Barium-Seeded Hydrogen Discharge. Phys. Rev. Lett., 62:764-767, 1989.

[6] J. Pamela. The physics of production, acceleration and neutralization of large negative ion beams. Plasma Phys. Control. Fusion, 37:A325-A336, 1995.

[7] P. Franzen et al. Physical and Experimental Background of the Design of the ELISE Test Facility. AIP Conf. Proc., 1097:451-460, 2008.

[8] B. Heinemann, H. D. Falter, U. Fantz, and P. Franzen et al. Design of the "half-size" ITER neutral beam source for the test facility ELISE. Fus. Eng. Des., doi:10.1016/j.fusengdes.2008.11.076, 2009.

[9] R. Gutser, D. Wünderlich, and U. Fantz et al. Negative hydrogen ion transport in RF-driven ion sources for ITER NBI. Plasma Phys. Control. Fusion, 51:045005-1-045005-14, 2009.

[10] P. McNeely, S. V. Dudin, S. Christ-Koch, and U. Fantz et al. A Langmuir probe system for high power RF-driven negative ion sources on high potential. Plasma Sources Sci. Technol., 18:014011-1-014011-17, 2009.

[11] M. Bacal, A. M. Bruneteau, C. Deniset, L. I. Elizarov, F. Sube, and A. Y. Tontegode. Effect of cesium and xenon seeding in negative hydrogen ion sources. Rev. Sci. Instr., 71:1082-1085, 2000.

[12] M. Čerček and T Gyergyek. PIC Simulation of a Double Layer Formation in a Hydrogen Plasma with Negative Ions. In Proc. 29th EPS Conference on Plasma Phys and Contr. Fusion, 2002.

[13] F. Taccogna, S. Longo, M. Capitelli, and R. Schneider. Negative-Ion-Source Modeling: From Expansion to Extraction Region. IEEE Trans. Plasma Sci., 36:1589-1599, 2008.

[14] M. Bandyopadhyay and R. Wilhelm. Simulation of negative hydrogen ion production and transport. Rev. Sci. Instrum., 75:1720-1722, 2004.

[15] R. F. Fernsler. Modeling Langmuir probes in multi-component plasmas. Plasma Sources Sci. Technol., 18:014012-1-014012-12, 2009.

[16] V. Dudnikov. Thirty years of surface plasma sources for efficient negative ion production. Rev. Sci. Instr., 73:992-994, 2002.

[17] O. Fukumasa. Modeling of negative ion production in hydrogen plasmas. IEEE Trans. Plasma Sci., 28:1009-1015, 2000.

[18] J. D. Isenberg, H. J. Kwon, and M. Seidl. Surface production of $\mathrm{H}^{-}$ions by backscattering of $\mathrm{H}^{+}$ and $\mathrm{H}_{2}^{+}$ions in the 3-50 eV ion energy range. AIP Conf. Proc., 287:38-47, 1994.

[19] B. Rasser, J. N. M. van Wunnik, and J. Los. Theoretical models of the negative ionization of hydrogen on clean tungsten, cesiated tungsten and cesium surfaces at low energies. Surf. Science, 118:697-710, 1982.

[20] W. G. Graham. In Proc. 2nd Int. Symp. on the Production and Neutralisation of Negative Hydrogen Ions and Beams, Upton, New York, 1980. Brookhaven National Laboratory.

[21] U. Fantz, P. Franzen, and W. Kraus et al. Negative ion RF sources for ITER NBI: status of the development and recent achievements. Plasma Phys. Control. Fusion, 49:B563-B580, 2007.

[22] R. F. Welton, M. P. Stockli, and S. N. Murray. Advances in the performance and understanding of the Spallation Neutron Source ion source. Rev. Sci. Instrum., 77:03A506-1-03A506-3, 2006.

[23] U. Fantz, H. D. Falter, P. Franzen, E. Speth, R. Hemsworth, and D. Boilson. Plasma diagnostic 
tools for optimizing negative hydrogen ion sources. Rev. Sci. Instrum., 77:03A516-1-03A516-4, 2006.

[24] B. S. Lee and M. Seidl. Surface production of $\mathrm{H}^{-}$ions by hyperthermal hydrogen atoms. Appl. Phys. Lett., 61:2857-2859, 1992.

[25] E. Eckstein and J. P. Biersack. Reflection of low-energy hydrogen from solids. Appl. Phys. A, $38: 123-129,1985$.

[26] E. Eckstein. Calculated Sputtering, Reflection and Range Values. Technical Report IPP 9/132, Max-Planck-Institut für Plasmaphysik, Garching, 2002.

[27] M. Bacal. Physics aspects of negative ion sources. Nucl. Fusion, 46:S250-S259, 2006.

[28] L. A. Schwager and C. K. Birdsall. Collector and source sheaths of a finite ion temperature plasma. Phys. Fluids B, 2:1057-1068, 1990.

[29] C. K. Birdsall and A. B. Langdon. Plasma Physics via Computer Simulation. McGraw-Hill, New York, 1985.

[30] http://www.nag.co.uk/.

[31] E. Kawamura, C. K. Birdsall, and V. Vahedi. Physical and numerical methods of speeding up particle codes and paralleling as applied to RF discharges. Plasma Sources Sci. Technol., 9:413$428,2000$.

[32] F. Taccogna, R. Schneider, S. Longo, and M. Capitelli. Modeling of a negative ion source. II. Plasma-gas coupling in the extraction region. Phys. Plasmas, 15:103502-1-103502-10, 2008.

[33] R. J. Procassini, C. K. Birdsall, and E. C. Morse. A fully kinetic, self-consistent particle simulation model of the collisionless plasmasheath region. Phys. Fluids B, 2:3191-3205, 1990.

[34] U. Fantz, H. D. Falter, P. Franzen, and D. Wünderlich et al. Spectroscopy - a powerful diagnostic tool in source development. Nucl. Fusion, 46:S297-S306, 2006.

[35] U. Fantz and D. Wünderlich. A novel diagnostic technique for $\mathrm{H}^{-}\left(\mathrm{D}^{-}\right)$densities in negative hydrogen ion sources. New J. Phys., 8:301-1-301-23, 2006.

[36] M. Berger et al S. Christ-Koch, U. Fantz. Laser photodetachment on a high power, low pressure rf-driven negative hydrogen ion source. Plasma Sources Sci. Technol., 18:025003-1-025003-12, 2009.

[37] S. Christ-Koch et al M. Berger, U. Fantz. Cavity ring-down spectroscopy on a high power rf driven source for negative hydrogen ions. Plasma Sources Sci. Technol., 18:025004-1-025004-8, 2009.

[38] J. D. Hobbs und J. A. Wesson. Heat flow through a Langmuir sheath in the presence of electron emission. Plasma Phys., 9:85-87, 1967.

[39] T. Intrator, M. H. Cho, E. Y. Wang, N. Hershkowitz, D. Diebold, and J. DeKock. The virtual cathode as a transient double sheath. J. Appl. Phys., 64:2927-2933, 1988. 